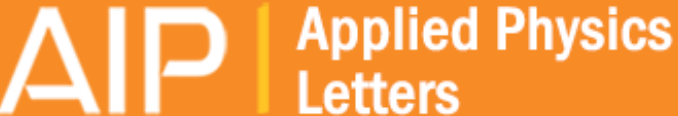

\section{THE PRESENCE OF DEEP LEVELS IN ION IMPLANTED JUNCTIONS}

R. G. Hunsperger, O. J. Marsh, and C. A. Mead

Citation: Applied Physics Letters 13, 295 (1968); doi: 10.1063/1.1652619

View online: http://dx.doi.org/10.1063/1.1652619

View Table of Contents: http://scitation.aip.org/content/aip/journal/apl/13/9?ver=pdfcov

Published by the AIP Publishing

\section{Articles you may be interested in}

A nitrogen-related deep level defect in ion implanted $4 \mathrm{H}-\mathrm{SiC}$ pn junctions-A spin dependent recombination study

Appl. Phys. Lett. 100, 112113 (2012); 10.1063/1.3695330

Deeplevel transient spectroscopy and electrical characterization of ionimplanted pn junctions into undoped InP

J. Appl. Phys. 78, 5325 (1995); 10.1063/1.359710

Deep levels related to ionimplanted tellurium in silicon

J. Appl. Phys. 54, 6417 (1983); 10.1063/1.331919

Deep levels in ionimplanted Si after beam annealing

Appl. Phys. Lett. 40, 68 (1982); 10.1063/1.92928

Deep levels in ionimplanted, CW laserannealed silicon

AIP Conf. Proc. 50, 550 (1979); 10.1063/1.31715

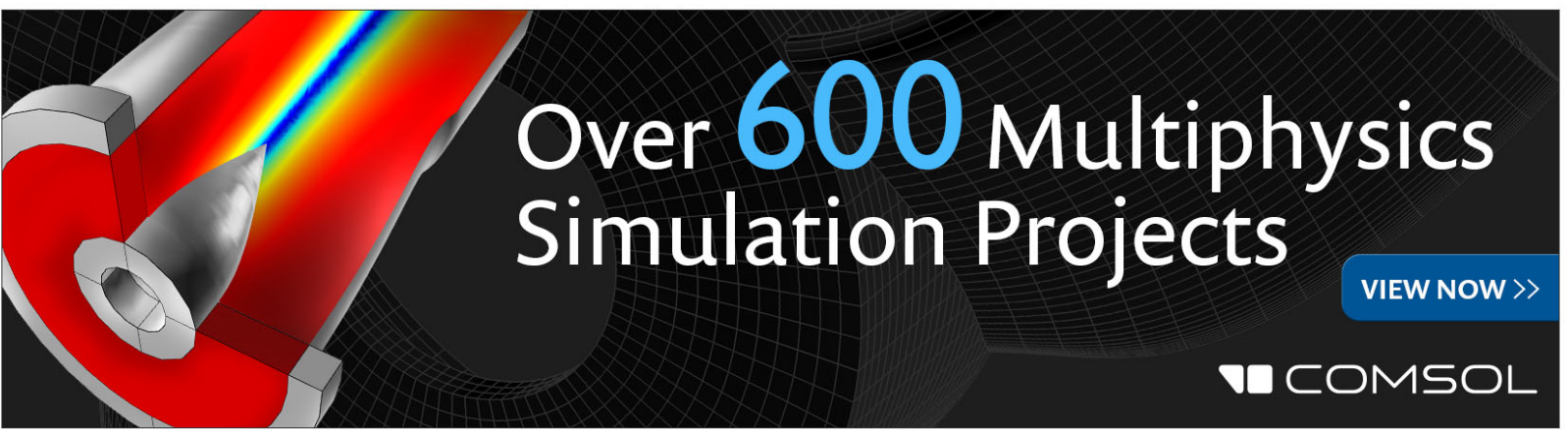


side, the built-in field of the junction would not retard their motion.

${ }^{10}$ R. K. Swank, M. Aven, and J. Z. Devine, J. Appl. Phys. (to be published).
${ }^{11}$ D. G. Thomas and E. A. Sadowski, J. Phys. Chem. Solids 25, 395 (1964).

${ }^{12}$ M. Aven and W. Garwacki, J. Appl. Phys. 38, 2302 (1967).

\title{
THE PRESENCE OF DEEP LEVELS IN ION IMPLANTED JUNCTIONS*
}

\author{
R. G. Hunsperger and O. J. Marsh \\ Hughes Research Laboratories, \\ Malibu, California 90265 \\ C. A. Mead \\ California Institute of Technology \\ Pasadena, California 91109
}

(Received 31 July 1968; in final form 24 September 1968)

\begin{abstract}
It has been found that ion implantation doping results in the generation and diffusion of defect species, forming deep trapping levels. The effect of these levels on the electrical characteristics of zinc-implanted GaAs diodes has been observed for the case of $70-\mathrm{kV}$ implantation at $400^{\circ} \mathrm{C}$ into substrates with $n$-type concentrations ranging from $1 \times 10^{16}$ to $1.8 \times 10^{18}$ atoms $/ \mathrm{cm}^{3}$. Capacitancevoltage measurements have indicated the presence of a semi-insulating layer in the diodes, varying in thickness from $0.18 \mu$ for the most heavily doped substrate to $2.7 \mu$ for the lightest. Frequency dependence of the junction capacitance and power law variation of forward current vs voltage have also been observed and are attributed to deep levels.
\end{abstract}

It is well known that the presence of deep levels in the energy gap of a semiconductor can result in the trapping of carriers, which leads to such phenomena as compensation and excess current in diodes. When a semiconductor is bombarded by a beam of highenergy particles, as it is in ion implantation doping, a layer of damage is produced which extends to a depth on the order of the projected range of the particles. ${ }^{1}$ Localized defects (vacancies and interstitials) produced by the bombardment may diffuse through the lattice to form more complex and stable defects at depths exceeding the projected range of the implanted ions. These defects can cause deep level states in the energy gap. Hence, one expects to see the effect of these states on the characteristics of ion-implanted devices, and indeed it has been observed in the case of zinc-implanted GaAs diodes.

The diodes used in these experiments were formed by implanting a dose of approximately $10^{15}-10^{16} / \mathrm{cm}^{2}$ singly ionized zinc ions at $70 \mathrm{kV}$ into $n$-type $\mathrm{GaAs}$ substrates held at $400^{\circ} \mathrm{C}$. The implantation apparatus has been described previously. ${ }^{2}$ After implantation the samples were annealed at $500^{\circ} \mathrm{C}$ for $5 \mathrm{~min}$ and were etched to produce mesa diodes approximately $0.5-\mathrm{mm}$ diameter. The annealing was necessary to reduce the resistivity of the implanted layer to a reasonable value. However, anneal time was kept as brief as possible and temperature as low as possible so that significant diffusion of the zinc would not take place.

Contact was made to the $p$-type side of the diode

* This work was supported in part by NASA-ERC, Cambridge, Mass. using indium solder, and to the $n$-type side with evaporated tin which was heat treated at $425^{\circ} \mathrm{C}$ for $2 \mathrm{~min}$.

The most interesting characteristic of these ionimplanted diodes was that they appeared to have a semi-insulating layer and resultant $p-i-n$ structure rather than the expected abrupt $p-n$ junction. This feature can be best seen from the capacitancevoltage characteristics for a number of typical diodes, shown in Fig. 1. Data are shown for implants made into substrates of four different $n$-type impurity concentrations. In every case $1 / C^{2}$ vs $V$ curve is offset from that which would be obtained for a normal $p-n$ junction. In Fig. 1(b) the curve for an evaporated aluminum surface barrier is given for comparison. This surface barrier was formed on a sample of the same substrate material as the implanted diode, after the substrate was subjected to all of the processing and annealing steps except for the actual implantation of ions. Thus the offset which was observed for the diode curve is a result of implantation rather than processing or heat treating. This offset corresponds to a decrease in junction capacitance, which we believe results from the formation of a semi-insulating layer in the junction. The amount of offset of the $1 / C^{2}$ vs $V$ curves from the curve expected for an abrupt junction was used to calculate the effective series capacitance and thus the thickness of the semi-insulating region in each case. The results of such calculations, which are given in Fig. 2, show that the semi-insulating region thickness depends on the substrate background doping concentration-varying approximately as the inverse of the square root of the concentration. Note that in all cases the thickness of 


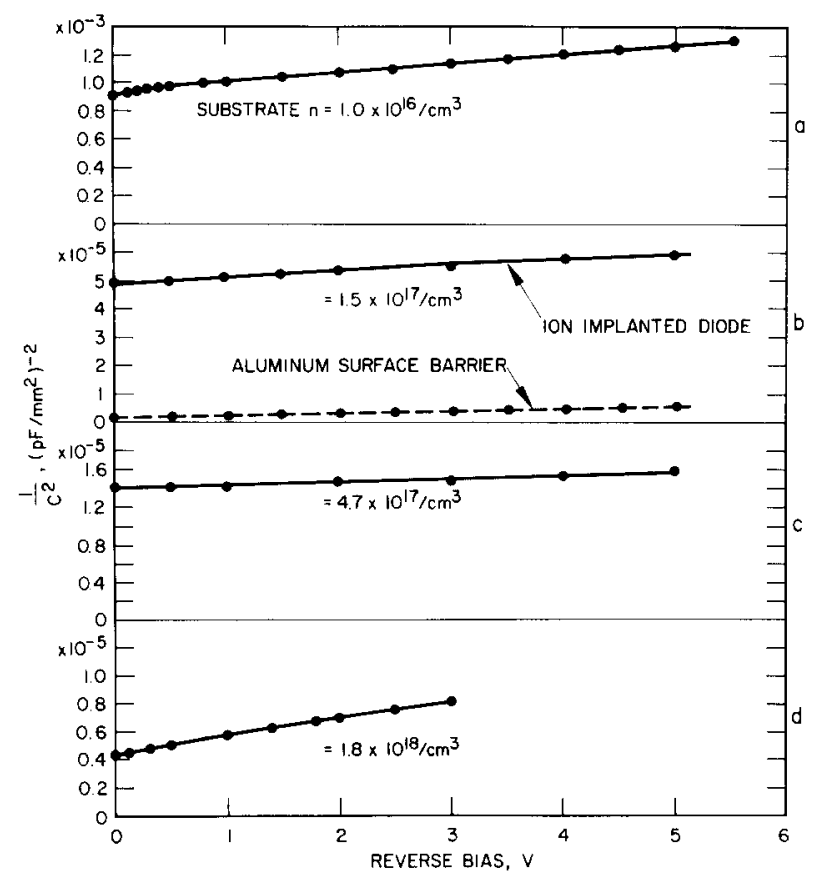

Fig. 1. Capacitance vs voltage characteristics for zinc-implanted GaAs diodes.

the semi-insulating layer far exceeds the theoretical Lindhard et $a l .^{3}$ range of the ions. It is very unlikely that channeled ions are responsible for the thick semi-insulating layer because no attempt was made to align the substrate with the beam and yet the thickness of the layer was consistent for various samples of the same substrate. (The beam alignment with the sample was close to the $\{1,1,1\}$ direction but varied about $\pm 3^{\circ}$ from sample to sample.)

For the temperatures and times used for implantation and annealing of these samples it is expected that zinc diffusion is negligible. It is believed that the relatively thick semi-insulating layer results from deep diffusing defects producing compensation to the depth where the concentration of defects equals the substrate doping concentration. Thus the data of Fig. 2 for diodes formed on substrates of different impurity concentrations represent a profiling of the defect concentration vs depth.

Another characteristic of these diodes which is indicative of the presence of deep level traps is the nonideal forward current vs voltage dependence, which follows an $I \alpha V^{n}$ relation, where $n=4$ to 5 . Such a power law dependence is expected for double injection of carriers into a semi-insulating region. The observed frequency dependence of the junction capacitance as shown in Fig. 3 is also attributable to deep levels. Data are given for two different substrate concentrations, and for two different temperatures in the case of the more lightly doped substrate. It can be seen that in all cases junction capacitance decreases with increasing frequency. This decrease can be explained by the fact that the

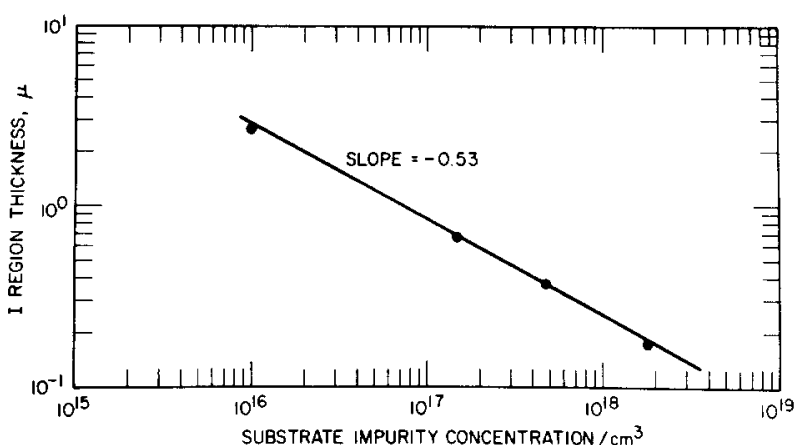

Fig. 2. Dependence of semi-insulating region thickness on substrate background impurity concentration.

defect trapping centers cannot charge and discharge rapidly enough to follow the applied signal at high frequencies, and thus cannot contribute to the capacitance. It should be noted that the capacitance measurements used previously to calculate the thickness of the semi-insulating layer were made at $500 \mathrm{kc} / \mathrm{sec}$, the highest available frequency, so that traps would not contribute appreciably to the capacitance and hence the true thickness of the semi-insulating region would be measured. From the results shown in Fig. 3 it can be seen that there was less frequency dependence of the capacitance for the sample formed on the more heavily doped substrate. Also, an increase in temperature resulted in larger capacitance at a given frequency, since the detrapping time was diminished. Such behavior of capacitance with frequency, doping and temperature is qualitatively in agreement with the behavior observed by Sah and Reddi ${ }^{4}$ for the case of deep levels in gold-doped silicon and with that observed by Schibli and Milnes ${ }^{5}$ for indium-doped silicon.

Detailed measurement of the temperature dependence of capacitance at $5 \mathrm{kc} / \mathrm{sec}$ indicated an exponential increase of capacitance with rising temperature, with a phenomenological activation energy of $0.29 \mathrm{eV}$.

The defects produced as a side effect of ion implantation are no doubt greatly affected by the particular implantation conditions. Work is presently in progress to establish the dependence on implant

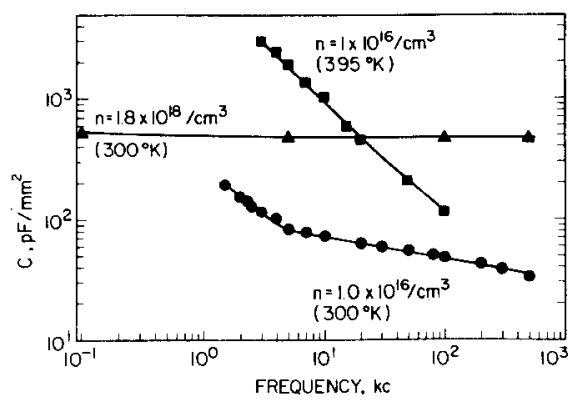

Fig. 3. Frequency dependence of junction capacitance for zinc-implanted GaAs diodes. 
temperature, voltage, ion dose, etc. and on subsequent annealing procedures.

It is clear that the generation and diffusion of species forming deep trapping levels is an inherent part of the implantation process and cannot be ignored when the presence of such levels may be important.

${ }^{1}$ P. Sigmund and J. Sanders, Conference on Application of Ion Beams to Semiconductor Technology,
Grenoble, France, May 1967.

${ }^{2}$ J. Mayer, O. Marsh, R. Mankarious, and R. Bower, J. Appl. Phys. 38, 1975 (1967).

${ }^{3}$ J. Lindhard, M. Scharff, and H. Schiфtt, Kgl. Danske Videnskab. Selskab, Mat. Fys. Medd. Dan. Vid. Selsk. 33, 1 (1963).

${ }^{4} \mathrm{G}$. Sah and V. Reddi, IEEE Trans. Electron Devices ED-11, 345 (1964).

${ }^{5}$ E. Schibli and A. Milnes, Solid State Electron. 11, 323 (1968).

\title{
ZEEMAN SPLITTING USED TO INCREASE ENERGY FROM A $Q$-SWITCHED IODINE LASER*
}

\author{
David W. Gregg, Ray E. Kidder, and Champe V. Dobler \\ Lawrence Radiation Laboratory, University of California, \\ Livermore, California 94550 \\ (Received 16 September 1968)
}

\begin{abstract}
Magnetic broadening of the iodine lasing line was found to increase the energy storage capability of the laser and thus increase the energy obtainable in a $Q$-switched pulse from a few millijoules to a few tenths of a joule.
\end{abstract}

The iodine laser, discovered by Kasper and Pimentel, ${ }^{1}$ has a great deal of potential for being developed into a high-power, $Q$-switched laser. The laser is pumped by photodissociating trifluoromethyliodide which forms excited atomic iodine.

$$
\mathrm{CF}_{3} \mathrm{I}+h \nu_{<2700 \AA} \rightarrow \mathrm{CF}+\mathrm{I}^{*} .
$$

The iodine then lases between the ${ }^{2} P_{1 / 2}$ and ${ }^{2} P_{3 / 2}$ states at $1.315 \mu$. Other fluoridated and unfluoridated, iodized carbon chains have been used with similar results. ${ }^{2,3}$ The principal problem in obtaining energetic $Q$-switched pulses from this laser is due to its inability to store large amounts of energy. When a laser is operated in a $Q$-switched fashion as opposed to a continuous or semicontinuous fashion, the energy obtained in the pulse has to be totally stored in the laser before lasing action begins. This is necessary since the pump source cannot deliver a significant amount of energy to the laser during the pulse. The storage limitation in this laser is caused primarily by the large cross section of the lasing transition. "A large cross section" means that for a relatively small amount of stored energy the laser will have a large gain. And, since the gain in the laser is limited to the point where it will lase spontaneously, which can happen even without mirrors, this limits the amount of energy that can be stored in the laser. This concept can be presented quantitatively as follows:

$$
\text { The gain in the laser }=G=e^{\alpha \Delta n L},
$$

where $\alpha$ is the cross section of the lasing line, $\Delta n$

*Work performed under the auspices of the U.S. Atomic Energy Commission. is the atomic inversion unit volume, and $L$ is the length of the laser.

The energy stored per unit frontal area

$$
=E=1 / 2 \Delta n h \nu L \text {, }
$$

where $h$ is Planck's constant and $\nu$ is the frequency of the laser light. A reasonable estimate for the maximum achievable gain in a laser is $10^{3}$. Slightly higher gains might be achievable, but somewhere close to this region superradiance will cause the laser to emit energy as rapidly as it can be pumped. If this number is substituted into Eq. (1), using Eq. (2), we find that:

$$
E_{\max }=3.5 h \nu / \alpha
$$

or the maximum energy storage capability of a laser per unit frontal area depends on the wavelength of the laser light and the cross section of the lasing line. A useful maximum energy density to achieve in a $Q$-switched pulse is $2 \mathrm{~J} / \mathrm{cm}^{2}$ of frontal area. If this is substituted into $\mathrm{Eq}$. (3), and letting $\lambda=1.315 \mu$, then $\alpha=2.6 \times 10^{-19} \mathrm{~cm}^{2}$. It is interesting to note that this is very close to the measured cross section for ruby and Nd glass lasers. However, a calculation of the cross section for the lasing transition in the iodine laser, assuming a Doppler-broadened line at room temperature gives, $\alpha=2.5 \times 10^{-17} \mathrm{~cm}^{2}$, which would allow the storage of only a few millijoules $/ \mathrm{cm}^{2}$ frontal area. We thus investigated the possibility of broadening the line with a magnetic field (Zeeman effect), which would lower the cross section and increase the energy storage capability of the laser. We found that, when a magnetic field is applied to the iodine, both the upper and lower lasing levels are split. ${ }^{4}$ This happens in such a way as to give six lines equally distributed around the original 